\title{
Unilateral lesions of the globus pallidus: report of four patients presenting with focal or segmental dystonia
}

\author{
A Münchau, D Mathen, T Cox, N P Quinn, C D Marsden, ^ K P Bhatia
}

\begin{abstract}
Objectives-To interpret clinical features after unilateral lesions of the globus pallidus on the basis of physiology of the basal ganglia.

Methods-Four patients with unilateral lesions in the globus pallidus (GP) were clinically examined and the literature on patients with pallidal lesions was reviewed.

Results-Three patients presented with contralateral dystonia largely confined to one arm in one case and one leg in two cases. One patient had predominant contralateral hemiparkinsonism manifested mainly as micrographia and mild dystonia in one arm. The cause of the lesions was unknown in two patients. In the other two symptoms had developed after head trauma and after anoxia. All lesions involved the internal segment of the GP. Two patients, including the patient with hemiparkinsonism, had additional involvement of the external segment of the GP. In the literature reports on 26 patients with bilateral lesions restricted to the GP only two with unilateral lesions were found. The patients with bilateral pallidal lesions manifested with dystonia, parkinsonism, or abulia. One of the patients with unilateral GP lesions had contralateral hemidystonia, the other contralateral arm tremor. Conclusion-These cases emphasise the importance of the GP, particularly its internal segment, in the pathophysiology of dystonia.

(F Neurol Neurosurg Psychiatry 2000;69:494-498)
\end{abstract}

Keywords: globus pallidus lesions; basal ganglia; dystonia

Motor and other effects produced by selective lesions affecting the basal ganglia may give clues to their function. Focal lesions restricted to the globus pallidus (GP), particularly unilateral, are rare. In a recent review of behavioural and motor consequences of focal lesions of the

13 March 2000

Accepted 11 May 2000

^Died 29 September 1998

Case summaries of the reported patients

\begin{tabular}{|c|c|c|c|c|c|}
\hline Case & $\begin{array}{l}\text { Age of } \\
\text { onset }\end{array}$ & Latency & Clinical findings & Lesion on MRI & Cause \\
\hline 1 & $22 \mathrm{y}$ & $3 \mathrm{y}$ & Dystonia $\mathrm{L}$ arm, clawing $\mathrm{L}$ toes & $\mathrm{R}$ dorsal GP & Head trauma \\
\hline 2 & 6 months & Unknown & Dystonia L leg & R GPi & Unknown \\
\hline 3 & $22 \mathrm{y}$ & Unknown & $\begin{array}{l}\mathrm{L} \text { hemiparkinsonism, dystonia } \mathrm{L} \\
\text { arm }\end{array}$ & R GPe and GPi & Unknown \\
\hline 4 & $5 y$ & $\begin{array}{l}\text { Several } \\
\text { months }\end{array}$ & Dystonia R foot & L GPi & Anoxia \\
\hline
\end{tabular}

$\mathrm{GP}=\mathrm{Globus}$ pallidus; $\mathrm{GPe}=$ external segment of the globus pallidus; $\mathrm{GPi}=$ internal segment of the gobus pallidus. basal ganglia in humans only 17 patients with lesions restricted to the GP were identified but in only two was the lesion unilateral. ${ }^{1}$ Since then only nine additional patients with bilateral pallidal lesion have been reported. ${ }^{2-4}$ Here we describe four patients with discrete unilateral lesions of the GP (table).

\section{Case reports}

CASE 1

At the age of 19 this right handed man was involved in a road traffic accident while driving. He was struck on the right side of his head and was comatose for 24 hours. On regaining consciousness he had both anterograde and retrograde amnesia for about a day. $\mathrm{He}$ also had double vision which receded after a few days, transient mild facial asymmetry on smiling, and difficulties in performing skilled movements with his left hand. Since his recovery he had experienced mild problems with short term memory, but was able to retain his job as an engineer. Three years after the accident he noted that his left arm started to involuntarily pull across his chest so that the fingers would touch his right shoulder. These spasms initially occurred for 5-10 seconds at a time and later became continuous. There was no alteration in the level of consciousness during these attacks and they were not associated with pain or altered sensation in the arm. When the arm hung loosely he felt the muscles tensing involuntarily. During spasms the fingers flexed at the metacarpophalangeal and extended at the interphalangeal joints. Occasionally he noted that his left big toe would claw independently. $\mathrm{He}$ had no other medical history and had no familial or personal history of psychiatric or movement disorders. There was no history of toxic exposure or treatment with neuroleptic drugs. On examination 3 years after the onset of his symptoms at the age of 25 he had some immediate recall problems but did not show other neuropsychological deficits or behavioural abnormalities. His face was slightly asymmetric at rest, but facial movements were symmetric. He had recurrent dystonic spasms of the left arm with internal rotation and adduction of the left arm, flexion of the elbow and wrist, and extension of the fingers. Dystonic activity was found mainly in the left pectoralis major, trapezius, biceps, triceps and brachioradialis muscles. Distal hand movements were well preserved.

$\mathrm{He}$ walked without swinging his left arm. The neurological examination was otherwise normal. Brain MRI (fig 1) done 7 years after his head injury showed a lesion in the dorsal 


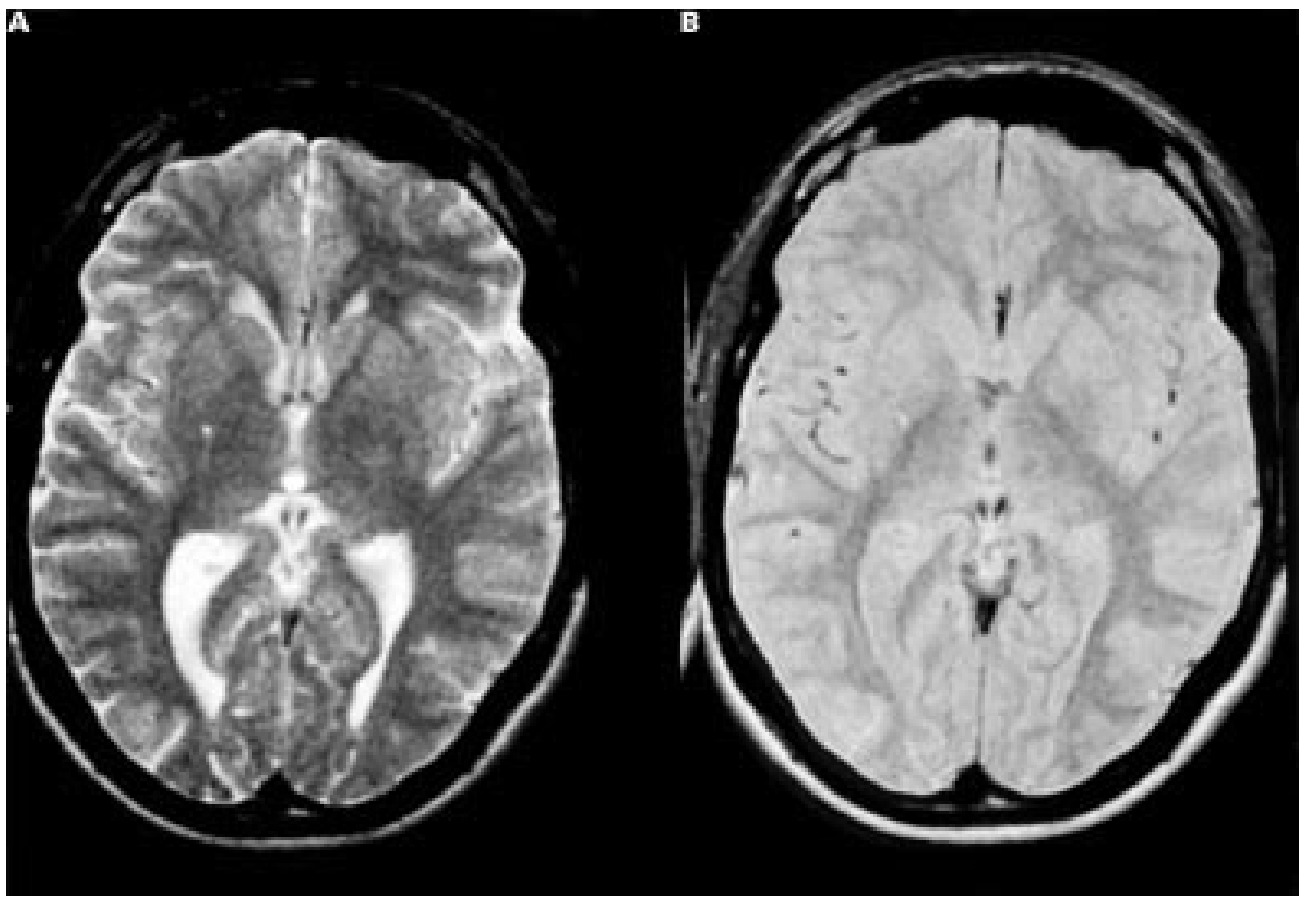

Figure 1 Brain MRI of case 1. (A) On the T2 weighted image and (B) the proton density image a small focus of hyperintensity is visible in the right dorsal GP.

part of the right GP. The lesion was hyperintense on T2 weighted and proton density images. Tetrabenazine, baclofen, clobazam, carbamazepine, and sodium valproate had no effect. His symptoms responded to higher doses of clonazepam but he had to reduce the dose due to aggression and irritability. $\mathrm{He}$ responded well to botulinum toxin injections into his left shoulder and upper arm muscles.

CASE 2

This 3 year old boy was the product of a normal pregnancy and delivery. There was no documented history of any perinatal problems, infections, head injury, drug exposure, or metabolic abnormalities. He had developed involuntary inversion of the left foot and flexion of the left knee and hip at the age of 6 months. He crawled from 8 months, stood at 10 months, walked at 17 months, and his walking was its best at about 2 years of age. His mental development was normal. He was first examined at the age of 3 years when severe dystonia of the left leg below the knee was noted. On examination at the age of 7 years the left foot was held in an equinovarus position and his leg was flexed at the hip and knee. He could straighten his leg to

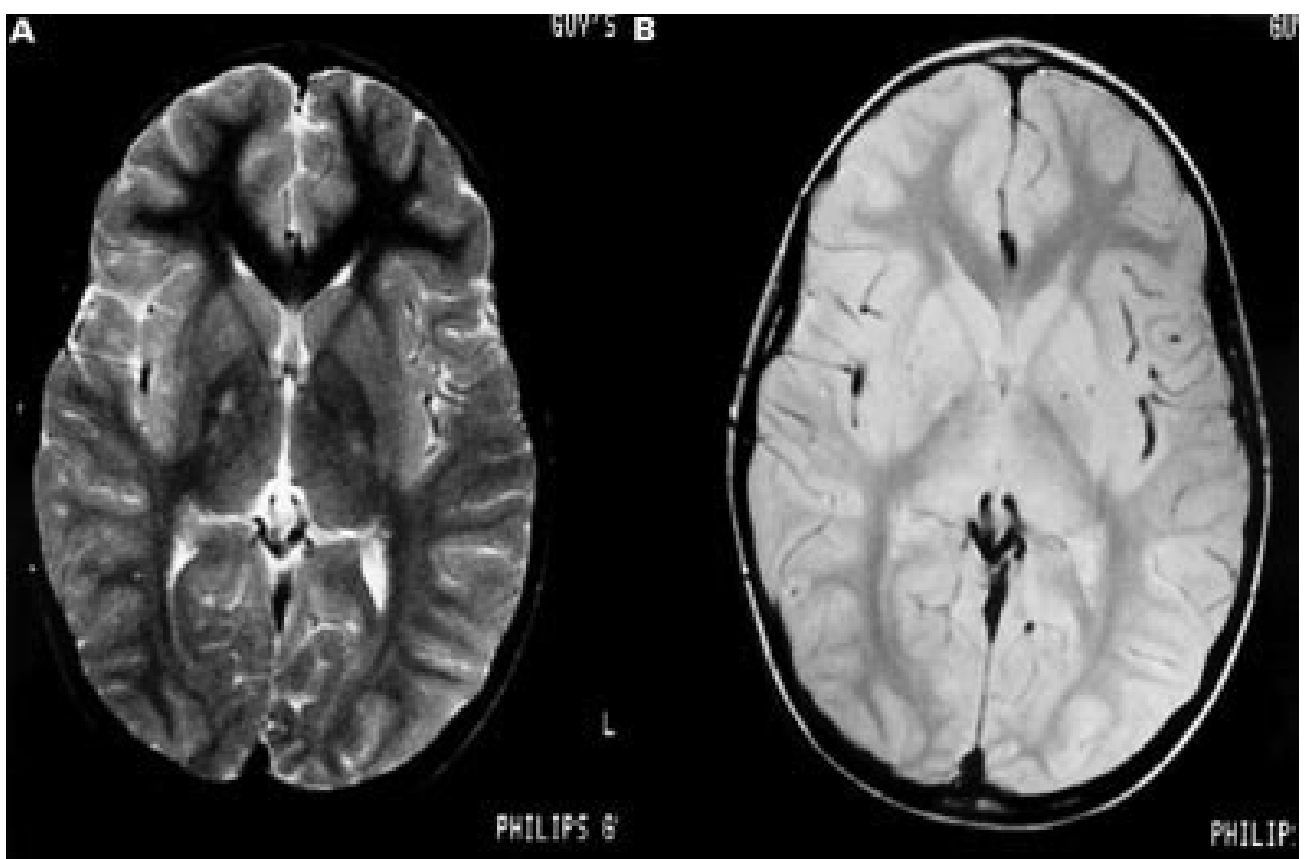

Figure 2 Brain MRI of case 2. (A) A small lesion in the internal segment of the right GP that is hyperintense on the T2 weighted image and $(B)$ the proton density image is shown. 


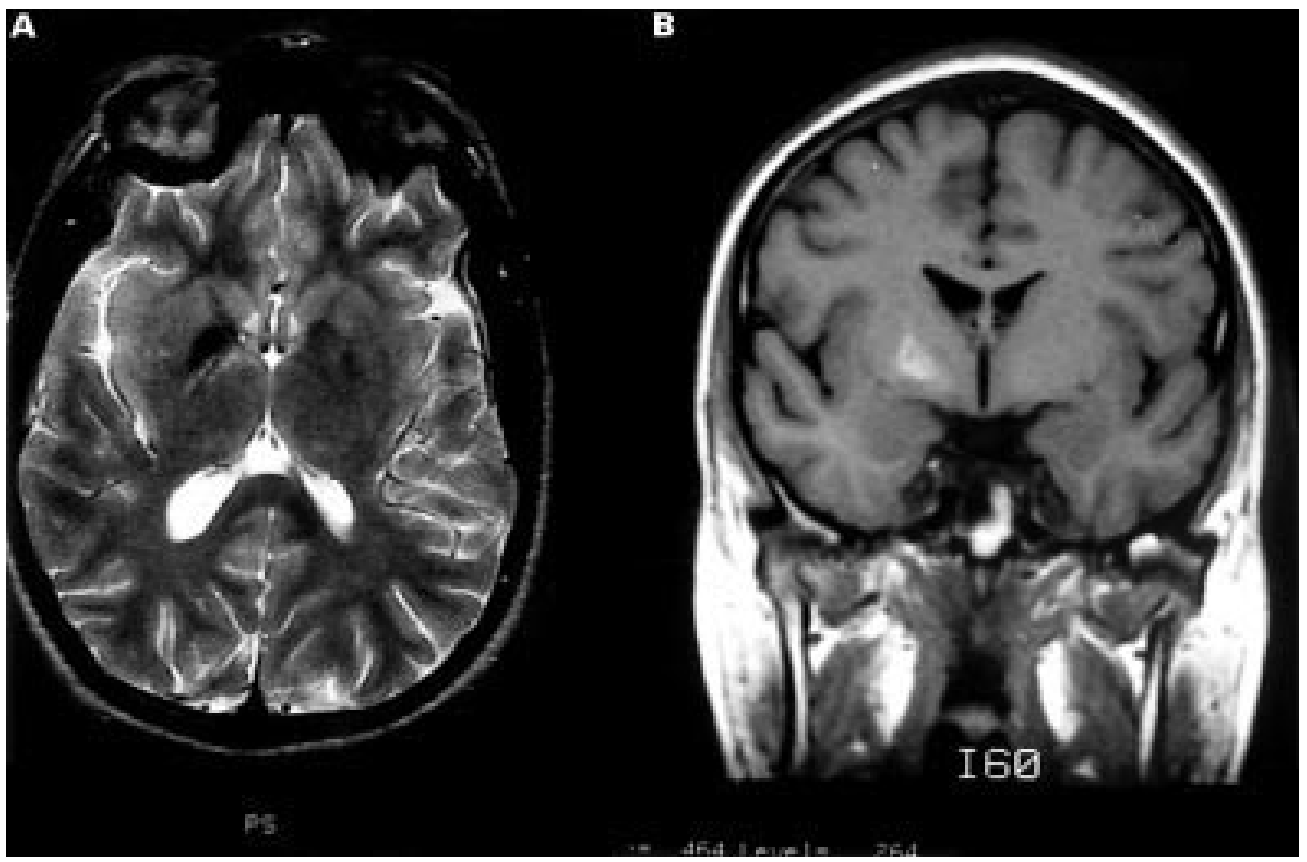

Figure 3 Brain MRI of case 3. An isolated lesion involving the internal and external segments of the right GP is shown. (A) The lesion is hypointense on the T2 weighted image and (B) hyperintense on the T1 weighted image. The coronal T1 weighted image (B) confirms the situation of the lesion and demonstrates patchy high signal consistent with microcalcification or mineralisation (blood degradation products): no evidence of calcification was seen at this site on CT.

bear weight. On passive manipulation the foot could be everted and inverted fully and dorsiflexed past 90 degrees. He could stand on one leg and walked with the help of crutches. The rest of the neurological examination was normal. He was of normal intelligence. Metabolic screening was negative. Brain MRI (fig 2) at the age of 7 years showed a small $(4 \mathrm{~mm})$ lesion in the internal segment of the right GP that was hyperintense on T2 weighted and proton density images, probably representing a small vascular lesion. Repeat MRI at the ages of 8,9 , and 12 years did not show any change. He was given adequate trials of levodopa, benzhexol, and baclofen without benefit.

CASE 3

This 27 year old left handed man presented with a 5 year history of difficulties with handwriting. He had noted that his writing was becoming progressively smaller. He had also noticed that his left ankle tended to invert on walking and neck muscles on the left tended to tense on and off, especially when writing. There was no significant medical history apart from asthma, no exposure to medication, and no family history of movement disorders. On examination there was slight hyperpronation of the left arm and some flexion and abduction of the little finger, but no other evidence of dystonia. He had marked micrographia and slowness of writing. In addition, there was akinesia and fatiguing of alternating finger and wrist movements on the left and also of ankle movements on that side. Power and reflexes were normal apart from brisk knee jerks and crossed adductors. Plantars were flexor. His arm swing was diminished on the left. Neurological examination was otherwise normal. Brain MRI (fig 3) done 5 years after the onset of his symptoms showed an isolated lesion in the right GP involving both the internal and external segment. A repeat study done a year later did not show any change. The lesion was hyperintense on T1 and hypointense on T2 weighted images. Brain CT was normal.

\section{CASE 4}

This patient had normal development until the age of 5 years when he had an operation on his left hip for an abscess. Apparently his heart stopped during this operation and he was in a coma for a few days. Several months after the operation he began to limp on his right leg. He was, however, able to walk reasonably well until the age of 15 years when he began to have cramps in the right calf associated with more intense spasms of the right foot with his toes forcibly flexing and the ankle twisting inwards. Symptoms did not spread to other body regions.

Medical history was otherwise unremarkable and there was no family history of movement disorders or other neurological disease. On examination at the age of 25 the patient walked with his right foot in an abnormal position with abduction of the toes. When lying on the bed any voluntary action of the left leg led to overflow dystonia in the right leg with the foot turning in and the toes plantar flexing. Tone was increased in the right leg. Tendon reflexes were variable, sometimes being brisker on the right leg than on the left which was interpreted as the result of dystonia in the right leg leading to reinforcement at the time of muscle contraction. Both plantar responses were flexor. Neurological examination was otherwise normal. Brain CT did not show any abnormalities. However, brain MRI (fig 4) showed a small isolated lesion in the internal segment of the left GP. 


\section{Discussion}

We have described four patients with unilateral lesions in the GP. Three presented with contralateral dystonia, largely confined to one arm in one case and a leg in two cases. One patient had contralateral hemiparkinsonism manifested mainly as micrographia and mild dystonia in one arm (case 3). In two cases the cause was unknown. In the other two, symptoms developed after head trauma and after anoxia respectively. In these patients onset was gradual and delayed which has also been reported in other cases of symptomatic dystonia. ${ }^{5-8}$ The reason for this is not clear. It has been suggested that a delayed onset is the result of slowly evolving aberrant sprouting of surviving neurons adjacent to the lesion ${ }^{9}$ or secondary retrograde degeneration or functional changes in deafferented neurons. ${ }^{10-12}$

Cases with isolated unilateral pallidal lesions are rare. In a recent meta-analysis of consequences of basal ganglia lesions ${ }^{1}$ only two such cases $^{5}{ }^{13}$ were documented. Pettigrew and Jankovic $^{5}$ reported on a patient who developed dystonia of his left foot with subsequent spread to the entire left leg, left arm, and left hand 4 years after closed head injury. He presumably had a vascular lesion in the right GP. Maki et $a l^{13}$ described the case of a 4 year old boy who fell to the ground while playing on a swing and developed a transient right hemiparesis that was followed 1 month later by tremor of the right arm that lasted for several weeks. On CT he had a lesion in the left GP involving both the internal and external segment.

Bilateral lesions confined to the GP are more common than unilateral lesions, ${ }^{1}$ which can be explained by the fact that the GP is vulnerable to systemic hypoxia and intoxications, especially carbon monoxide poisoning. ${ }^{14-17}$ Patients with bilateral GP lesions can present with generalised dystonia or parkinsonism, sometimes associated with behavioural problems, particularly abulia or disinhibition. . $^{1-4-1618} 19$

What is the pathophysiology of symptoms occurring after unilateral or bilateral pallidal lesions? On the basis of the current basal ganglia model, data on animal studies, and clinical observations, Bhatia and Marsden ${ }^{1}$ concluded that lesions of the GPe might cause parkinsonism due to disinhibition of the subthalamic nucleus and the GPi with subsequent overinhibition of the ventroanterior and ventrolateral thalamus and hence the premotor cortex. Support for this comes from the finding of bilateral lesions confined to the GPe causing parkinsonian symptoms. ${ }^{2}$ This could explain why the pallidal lesions in case 3 that involved the GPe, gave rise to hemiparkinsonism in this patient.

Involvement of the GPi seems to be crucial to the dystonia present in all our patients. In all these cases the pallidal lesions affected the GPi, albeit not exclusively. Similarly, in their study of patients with bilateral pallidal lesions Bucher et al encountered dystonic symptoms, particularly in patients with lesions in the GPi.

Based on functional imaging data, an underactivity of the GPi releasing the ventral tier thalamic nuclei from their normal pallidofugal inhibition is also emphasised in current pathophysiological models of primary dystonia. ${ }^{20} 21$ Recordings of neuronal activity in GP during pallidotomy in patients with dystonia have recently shown lowered mean discharge rates, increased responses to peripheral manipulation (proprioceptive stimulation), and irregularly grouped discharges. ${ }^{22-25}$ Decreased discharge rates in both segments of the GP were interpreted as being caused primarily by overactive inhibitory projections from the striatum to both the GPi and the GPe via the direct and indirect pathway, respectively. ${ }^{23} 24$ Decreased activity in the GPe would lead to disinhibition of the subthalamic nucleus and

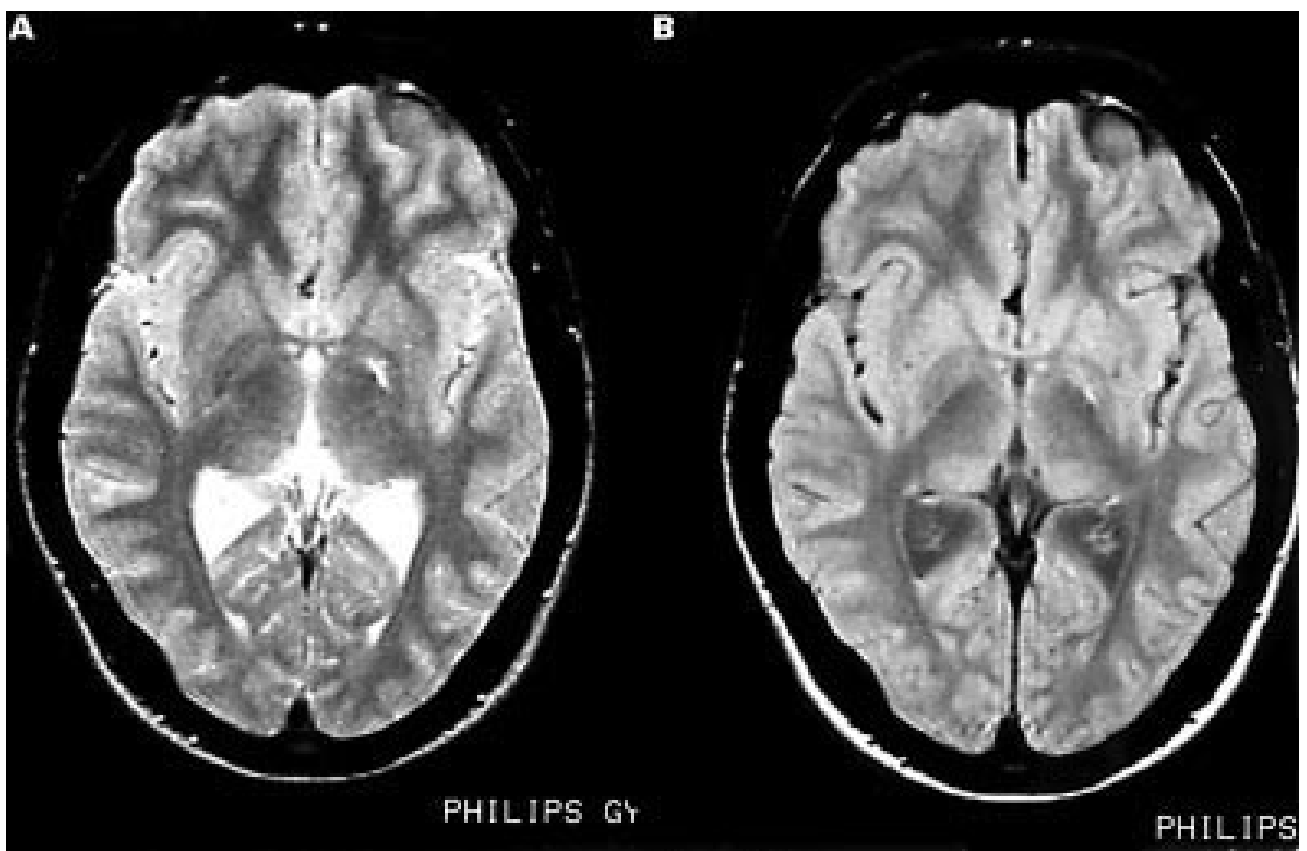

Figure 4 Brain MRI of case 4. (A) On the T2 weighted image and (B) the proton weighted image a small cleft like signal change is visible in the left Gpi. Part of this lesion does not have CSF signal intensity in the proton density image, which suggests that this area represents gliosis. 
thus to an increased drive from this nucleus to the GPi which could explain the increased response to proprioceptive stimuli in $\mathrm{Gpi}^{23}{ }^{24}$ as proprioceptive inputs reach the GPi probably largely via the subthalamic nucleus. ${ }^{26}$ However, the irregularities in pallidal activity may be more critical in the pathophysiology of idiopathic dystonia than the change of mean discharge rate or increased response to peripheral stimuli. ${ }^{24}{ }^{25}$ This may also apply to our cases of symptomatic dystonia due to pallidal lesions as these lesions are likely to disturb pallidal function, giving rise to irregular pallidal output, rather than abolish pallidal activity. This would explain the apparent paradox that a pallidal lesion in previously healthy subjects causes dystonia but GPi pallidotomy alleviates symptoms in patients with dystonia ${ }^{23-25}{ }^{27-29}$ as the first makes the GPi "noisy", the second, on the other hand, removes this "noise" by abolishing the output of an abnormally active GPi and may thereby lead to a normalisation of basal ganglia function. Alternatively, the consequences of a lesion within the GPi, whether pathological as in symptomatic dystonia or iatrogenic after pallidotomy, might be determined by the precise lesion location. For instance, apart from patient 1 , who had a lesion in the dorsal GPi, pallidal lesions in our patients seemed to be located anteriorly, whereas pallidotomy lesions in patients with dystonia are situated more dorsally. ${ }^{24} 27$

Symptomatic hemidystonia and also focal limb dystonia is most commonly associated with lesions of the contralateral putamen, ${ }^{158}$ perhaps because such lesions potentially affect both direct and indirect pathways ${ }^{1}$ which seems to be relevant for the development of dystonia. ${ }^{20}$ Although symptomatic hemidystonia is most commonly caused by putaminal lesions, the report of our four patients illustrates that contralateral dystonia can also occur in patients with pallidal lesions and confirms the notion that altered pallidal function, particularly the GPi, is an important element in the pathophysiology of acquired dystonia.

Dystonia was largely restricted to one limb in our patients, which may suggest a somatotopic representation within the GPi. In monkeys De Long et $a l^{30}$ could document some somatotopic organisation of the GPi but were unable to demonstrate any simple somatotopic representation of the different portions of the limbs (proximal and distal) within the arm or leg areas of the GPi. In a recent clinicoradiological study of five patients with striatopallidal lesions the distribution of symptoms also implied some somatotopy of the pallidum but precise characterisation was not possible. ${ }^{8}$ As only limited data on unilateral pallidal lesions in humans are available and localisation of lesions within the GP is still relatively imprecise, the precise somatotopic organisation of the GP in humans remains uncertain.

Finally, although visible lesions were confined to the globus pallidus in all our patients the history of coma in two (cases 1 and 4) and mild cognitive abnormalities in one (case 1) are suggestive of cerebral damage extending beyond the basal ganglia in these patients. This might have contributed to the development of symptoms.

AM was supported by the Ernst Jung-Stiftung für Wissenschaft und Forschung in Hamburg, Germany and the Eugene Brehm Bequest, United Kingdom.

1 Bhatia KP, Marsden CD. Behavioural and motor consequences of focal lesions of the basal ganglia in man. Brain quences of focal lesid

2 Bucher SF, Seelos KC, Dodel RC, et al. Pallidal lesions: structural and functional magnetic resonance imaging. Arch Neurol 1996;53:682-6.

3 Piccirilli M, Mazzi P, Luccioli R, et al. Selective bilateral lesion of the globus pallidus: 10 year follow up of memory impairment and frontal symptomatology. Ital F Neurol Sci 1995;16:635-40

4 Gruber Gikovate C, Zirretta JC, Ferreira Bezerra JM, et al. Transient globus pallidus T1 shortening associated with polycythaemia and dystonia. Neuroradiology 1999;41:28891.

5 Pettigrew LC, Jankovic J. Hemidystonia: a report of 22 patients and review of the literature. 7 Neurol Neurosurg Psychiatry 1985;48:650-7.

6 Marsden CD, Obeso JA, Zarranz JJ, et al. The anatomical Marsden CD, Obeso JA, Zarranz JJ, et al. The anatomical
basis of symptomatic hemidystonia. Brain 1985;108:46383.

7 Lehéricy S, Vidailhet M, Dormont D, et al. Striatopallidal and thalamic dystonia. A magnetic resonance imaging anatomoclinical study. Arch Neurol 1996;53:241-50.

8 Krystkowiak P, Martinat P, Defebvre L, et al. Dystonia after striatopallidal and thalamic stroke: clinicoradiological correlations and pathophysiological mechanisms. If Neurol Neurosurg Psychiatry 1998;65:703-8.

9 Burke RE, Fahn S, Gold AP. Delayed-onset dystonia in patients with 'static' encephalopathy. F Neurol Neurosurg Psychiatry 1980;43:789-97.

10 Miwa H, Hatori K, Kondon T, et al. Thalamic tremor. Neurology 1996;46:75-9.

11 Martin JP. Hemichorea (hemiballism) without lesions in the corpus luysii. Brain 1957; 80:101-5.

12 Dooling EC, Adams RD. The pathological anatomy of posthemiplegic athetosis. Brain 1975;98:29-48.

13 Maki Y, Akimoto H, Enomoto T. Injuries of basal ganglia following head trauma in children. Childs Brain 1980;7: $113-23$.

14 Klawans HL,Stein RW, Tanner CM, et al. A pure parkinsonian syndrome following acute carbon monoxide intoxication. Arch Neurol 1982;39:302-4.

15 Ali-Cherif A, Royere ML, Gosset A, et al. Behaviour and mental activity disorders after carbon monoxide poisoning. Bilateral pallidal lesions. Rev Neurol 1984;140:401-5.

16 Grinker RR. Parkinsonism following carbon monoxide poisoning. F Nerv Ment Dis 1926;64:18-28.

17 Hawker K, Lang AE. Hypoxic-ischemic damage of the basal ganglia. Case report and review of the literature. Mov Disord 1990;5:219-24.

18 Laplane D, Levasseur M, Pillon B, et al. Obsessivecompulsive and other behavioral changes with bilateral basal ganglia lesions. Brain 1989;112:699-725.

19 Jellinger K. Exogenous lesions of the pallidum. In:Vinken PJ, Bruyn GW, Klawans HL, eds. Handbook of clinical neuPJ, Bruyn GW, Klawans HL, eds. Handbook of clinical
rology. Amsterdam: Elsevier Science, 1986:465-91.

20 Crossman AR, Brotchie JM. Pathophysiology of dystonia. Adv Neurol 1998;78:19-25.

21 Eidelberg D. Metabolic brain networks in idiopathic torsion dystonia. Adv Neurol 1998;78:127-33.

2 Lenz, FA, Suarez JI, Verhagen Metman L, et al. Pallidal activity during dystonia: somatosensory reorganisation and changes with severity. If Neurol Neurosurg Psychiatry 1998;65:767-70

23 Vitek JL, Zhang J, Evatt M, et al. GPi pallidotomy for dystonia: clinical outcome and neuronal activity. $\mathrm{Adv} \mathrm{Neu}-$ rol 1998:78:211-9.

24 Vitek JL, Chockkan V, Zhang JY, et al. Neuronal activity in the basal ganglia in patients with generalised dystonia and hemiballismus. Ann Neurol 1999;46:22-35.

25 Lozano AM, Kumar R, Groass RE, et al. Globus pallidus internus pallidotomy for generalized dystonia. Mov Disord internus pallidot

26 Hamada I, DeLong MR. Excitotoxic acid lesions of the primate subthalamic nucleus result in reduced pallidal neuronal activity during active holding. F Neurorphysiol 1992;68: 1859-66.

27 Ondo WG, Desaloms JM, Jankovic J, et al. Pallidotomy for generalised dystonia. Mov Disord 1998;13:693-8.

28 Lin JJ, Lin GY, Shih C, et al. Benefit of bilateral pallidotomy in the treatment of generalised dystonia. Case report. $\mathcal{f}$ Neurosurg 1999;90:974-6.

29 Iacono RP, Kuniyoshi SM, Lonser RR, et al.. Simultaneous bilateral pallidoansotomy for idiopathic dystonia musculorum deformans. Pediatr Neurol 1996;14:145-8.

30 DeLong MR, Crutcher MD, Georgopoulos AP. Primate globus pallidus and subthalamic nucleus functional organization. F Neurophysiol 1985;53:530-43. 